\title{
Fabrication of Si nanopowder and application to hydrogen generation and photoluminescent material
}

\author{
Yuki Kobayashi, Kentaro Imamura, \\ Taketoshi Matsumoto, Hikaru Kobayashi ${ }^{*}$
}

\begin{abstract}
Si nanopowder is fabricated using the simple beads milling method. Fabricated Si nanopowder reacts with water in the neutral $\mathrm{pH}$ region between 7 and 9 to generate hydrogen. The hydrogen generation rate greatly increases with $\mathrm{pH}$, while $\mathrm{pH}$ does not change after the hydrogen generation reaction. In the case of the reactions of Si nanopowder with strong alkaline solutions (eg pH13.9), $1600 \mathrm{~mL}$ hydrogen is generated from $1 \mathrm{~g}$ Si nanopowder in a short time (eg $15 \mathrm{~min})$. When Si nanopowder is etched with HF solutions and immersed in ethanol, green photoluminescence (PL) is observed, and it is attributed to band-to-band transition of Si nanopowder. The Si nanopowder without HF etching in hexane shows blue PL. The PL spectra possess peaked structure, and it is attributed to vibronic bands of 9,10-dimethylantracene (DMA) in hexane solutions. The PL intensity is increased by more than 3,000 times by adsorption of DMA on Si nanopowder.
\end{abstract}

K e y w o r d s: beads milling, hydrogen generation rate, PL enhancement, adsorption

\section{Introduction}

Silicon nanopowder attracts much interest because of its properties largely different from Si bulk, $e g$ higher reactivity [1] and wider band-gap [2-3] due to the quantum confinement effect $[4,5]$. Various methods have been developed to fabricate Si nanopowder, eg plasma-enhanced chemical vapor deposition (PECVD) [6], laser ablation [7], and arc-discharge [8]. These methods require expensive materials such as $\mathrm{SiH}_{4}$, and moreover, the fabrication rate of $\mathrm{Si}$ nanopowder is considerably low. The $\mathrm{Si}$ nanopowder can also be produced using chemical methods, eg reduction of $\mathrm{SiCl}_{4}$ using eg $\mathrm{C}_{8} \mathrm{~K}$ [9], and $\mathrm{Mg}_{2} \mathrm{Si}$, [10]. The chemical method are also time-consuming, and requires expensive reactant materials.

We have developed a simple method to produce $\mathrm{Si}$ nanopowder by use of the beads milling method [11-15]. Produced Si nanopowder can be applied to solar cells [11], hydrogen generation material [12], anode material in Li ion batteries [13], and light emitting material [14-15].

Hydrogen evolution from water has been extensively studied so far mainly in order to solve the energy and environmental problems. Many researchers aimed to split water by use of photocatalysts such as $\mathrm{TiO}_{2}$. However, $\mathrm{TiO}_{2}$ is a wide-gap semiconductor, and thus, only ultraviolet light is usable, leading to low conversion efficiencies and thus to low hydrogen generation rates [16-17]. Several semiconductors such as $\mathrm{Ta}_{3} \mathrm{~N}_{5}$ which semiconductors absorb visible light have been developed for water splitting [18], but the hydrogen generation rate is still low. Another method to generate hydrogen uses irreversible chemical reactions, $e g$ the reaction of $\mathrm{Si}$ nanopowder with water.
In the previous studies, strong alkaline solutions are employed to generate hydrogen with high evolution rates [1], [19-20].

In the present study, hydrogen generation by the reaction of Si nanopowder with water in the neutral $\mathrm{pH}$ region, ie $\mathrm{pH} 7 \sim 9$, has been studied. Our finding is expected to be applied to medical use of Si nanopowder, ie internal hydrogen generation to eliminate hydroxyl radicals which cause various diseases such as Alzheimer's disease [21], Parkinson's disease [22], Huntington's disease [23], etc.

Si nanopowder emits photoluminescence (PL) in the visible region for several reasons. One of the reasons is due to band-gap widening of Si nanopowder arising from the quantum confinement effect [4-5]. In this case, the PL energy strongly depends on the size of Si nanopowder [24]. On the other hand, PL emission whose energy does not depend on the size of $\mathrm{Si}$ nanopowder is attributed to $\mathrm{SiO}_{2}$ interface states [25-26]. Size-independent PL may also arise from adsorption on Si nanopowder, ie PL from adsorbed species [27] or modification of PL energies by adsorption [28].

In the present study, we have observed two kinds of PL, ie green PL and blue PL, from Si nanopowder. The green PL energy strongly depends on the size of Si nanopowder and thus it is attributed to band-to-band transition of Si nanopowder. The blue PL energy does not depend on the size and adsorbed species exhibits PL with the great intensity enhancement.

* Institute of Scientific and Industrial Research, Osaka University, 8-1 Mihogaoka, Ibaraki, Osaka 567-0047, Japan, h.kobayashi@sanken.osaka-u.ac.jp 


\section{Experiments}

Two kinds of starting materials were used to produce Si nanopowder, (i) - commercial Si powder with $5 \mu \mathrm{m}$ size (Koujundo Chemical Laboratory Si 3N Powder), and (ii) - Si swarf generated during slicing Si ingots to produce Si wafers for solar cell use [11-15]. Si nanopowder was fabricated by the beads milling method. For the onestep beads milling method, $0.5 \mathrm{~mm}$ diameter zirconia beads were employed while for two-step beads milling, $0.3 \mathrm{~mm}$ diameter zirconia beads were used after milling with $0.5 \mathrm{~mm}$ diameter beads. In some cases, fabricated $\mathrm{Si}$ nanopowder was etched with $5 \mathrm{wt} \%$ hydrofluoric acid (HF) aqueous solutions to remove $\mathrm{SiO}_{2}$. For adjustment of $\mathrm{pH}$ of the solutions, $\mathrm{KOH}$ or borate buffer was employed.

Hydrogen concentrations in water were measured using a TOA DKK-TOA DH-35A potable dissolved hydrogen-meter. X-ray diffraction measurements were performed by use of a Rigaku RINT-2500 diffractometer. X-ray photoelectron spectroscopy (XPS) spectra were recorded using a KRATOTOS AXIS-165x spectrometer with an $\mathrm{Mg} \mathrm{K} \alpha$ radiation source.

For producing green PL-emitting Si nanopowder, asfabricated Si nanopowder was etched in $5 \mathrm{wt} \% \mathrm{HF}$ aqueous solutions, and then it was immersed in ethanol. The Si nanopowder without HF etching, on the other hand, showed blue PL after immersion in hexane. PL spectra were recorded with a JASCO FP-8500 spectrometer.

\section{Results and discussion}

\subsection{Hydrogen generation by reactions of Si nanopowder with water}

Figure 1(a) shows the XRD patterns of Si nanopowder observed after one-step beads milling of $5 \mu \mathrm{m}$ Si powder. All the observed peaks were attributable to Si crystals, indicating that Si nanopowder fabricated using the beads milling method possessed crystalline nature. The $\mathrm{Si}(111)$ peak had the highest intensity while no (100) peak was present due to the selection rule for the diamond crystal structure. Figure 1(b) shows the distribution of crystallite sizes of Si nanopowder obtained from the analysis of the $\mathrm{Si}(111)$ XRD peak. The average size and the mode diameter (ie the diameter at the maximum distribution) of Si nanocrystals was estimated to be 220 , and $210 \mathrm{~nm}$, respectively, before milling, 20.3 and $6.6 \mathrm{~nm}$, respectively, after one-step milling, and 12.2 and $5.8 \mathrm{~nm}$, respectively after two-step milling.
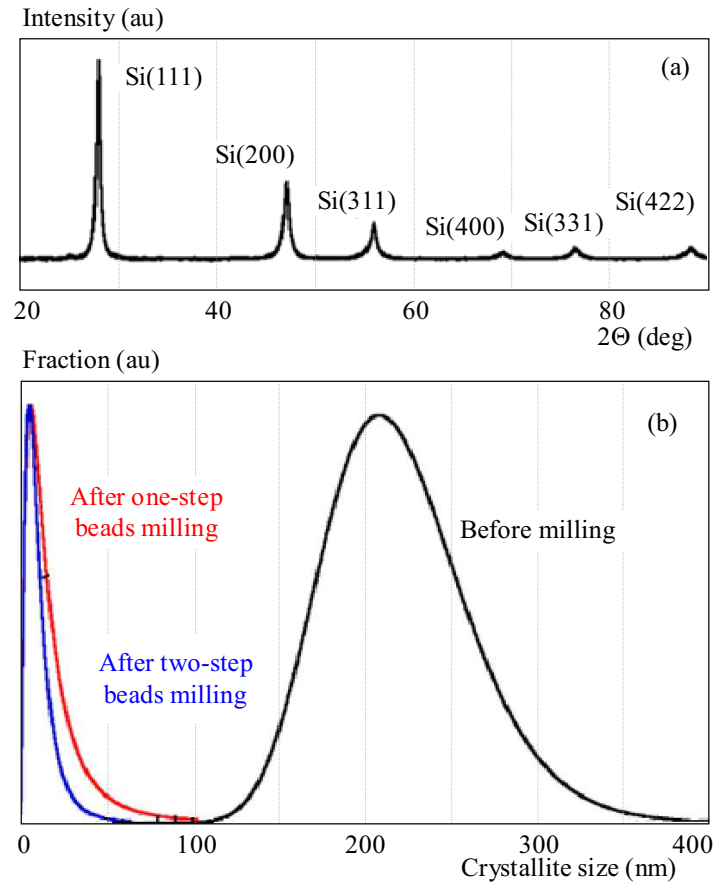

Fig. 1. XRD pattern for Si nanopowder (a) - produced with the one-step beads milling method, (b) - size distribution of Si powder before and after one-step and two-step beads milling

Figure 2 shows the hydrogen volume produced by the reaction of $\mathrm{Si}$ nanopowder with water vs. the reaction time. The hydrogen generation was carried out by addition of HF-etched $10 \mathrm{mg}$ Si nanopowder in $30 \mathrm{~mL}$ water at room temperature. For the solution with $\mathrm{pH}$ 8.0, borate buffer was added to ultrapure water. Even in the case of ultrapure water (curve a), hydrogen generation proceeded but the generation rate was very low.

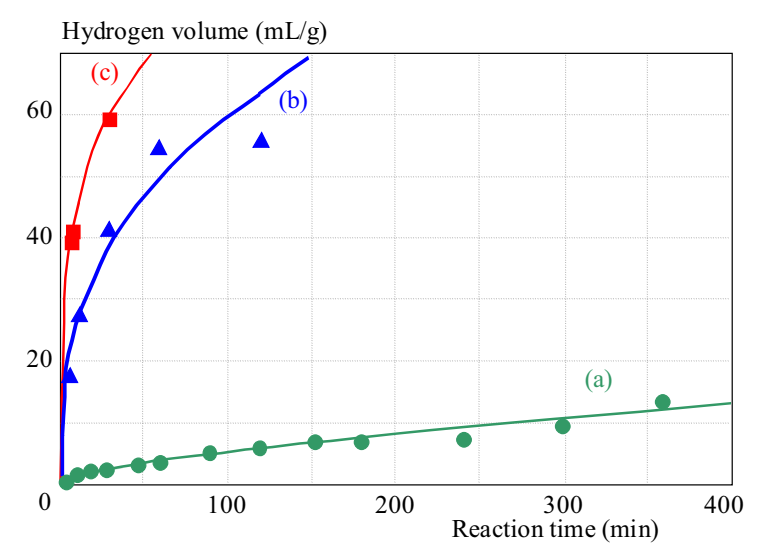

Fig. 2. Generated hydrogen volume $v s]$; the reaction time for HFetched $1 \mathrm{~g}$ Si nanopowder in water having following $\mathrm{pH}$ at room temperature: (a) - pH 7.0, (b) - pH 8.0, (c) - pH 9.0

The hydrogen generation rate increased by more than 20 times by an increase in $\mathrm{pH}$ to 8.0 (curve b). The hydrogen generation rate further increased by an increase in $\mathrm{pH}$ to 9.0 (curve c), and in this case, it was found that $\mathrm{pH}$ of the solution adjusted by $\mathrm{KOH}$ did not change after the hydrogen generation reaction. From these results, the 


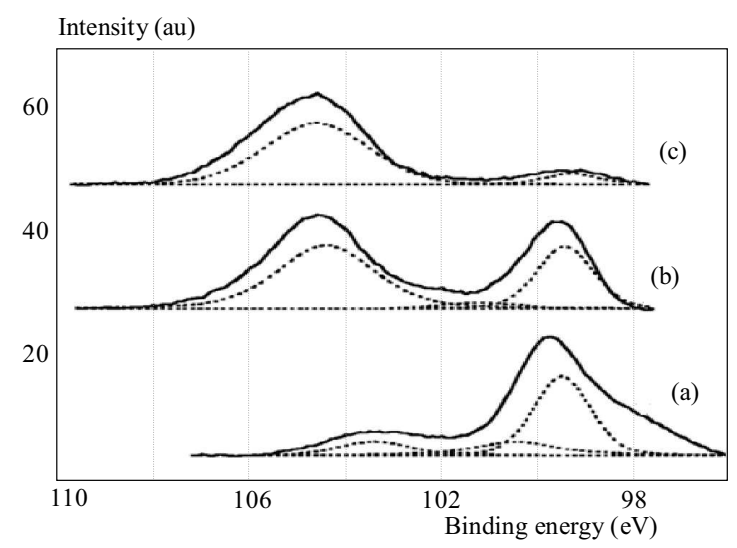

Fig. 3. XPS spectra of Si nanopowder observed after hydrogen generation by the reaction with water $(\mathrm{pH} 8.0)$ for the following periods: (a) $-10 \mathrm{~min},(\mathrm{~b})-3 \mathrm{~h},(\mathrm{c})-24 \mathrm{~h}$

most probable reaction formulae for hydrogen generation from Si nanopowder are expressed as

$$
\begin{gathered}
\mathrm{Si}+2 \mathrm{OH}^{-} \rightarrow \mathrm{H}_{2}+\mathrm{SiO}_{2}+2 e \\
2 \mathrm{H}_{2} \mathrm{O}+2 e \rightarrow \mathrm{H}_{2}+2 \mathrm{OH}^{-}
\end{gathered}
$$

Reaction (1) is the rate-determining step, and therefore, the reaction rate greatly increases with the concentration of $\mathrm{OH}^{-}$ions, ie $\mathrm{pH}$ of solutions. In reaction (1), $\mathrm{Si}$ nanopowder reacts with $\mathrm{OH}^{-}$ions, resulting in generation of hydrogen, $\mathrm{SiO}_{2}$, and electrons in the $\mathrm{SiO}_{2}$ conduction band. In reaction (2), electrons in the $\mathrm{SiO}_{2}$ conduction band are accepted by water molecules, leading to generation of hydrogen and $\mathrm{OH}^{-}$ions. Consequently, after the following overall reaction, ie reaction (1) plus reaction (2), the concentration of $\mathrm{OH}^{-}$ions is unchanged

$$
\mathrm{Si}+2 \mathrm{H}_{2} \mathrm{O} \rightarrow \mathrm{SiO}_{2}+2 \mathrm{H}_{2}
$$

It should be noted that in reaction (3), $\mathrm{OH}^{-}$ions act as catalyst. Figure 3 shows the XPS spectra in the Si $2 p$ region for Si nanopowder observed after the reaction with water (pH8.0). After the reaction for 10 min (spectrum a), a strong peak and a weak peak were observed and they were attributed to $\mathrm{Si}$ and $\mathrm{SiO}_{2}$, respectively [29-30]. After the reaction for $3 \mathrm{~h}$, the intensity of the peak due to $\mathrm{SiO}_{2}$ greatly increased (spectrum b). When the hydrogen generation reaction stopped, the intensity due to $\mathrm{Si}$ considerably decreased (spectrum c). The thickness of the $\mathrm{SiO}_{2}$ layer was estimated from the intensity ratio between the $\mathrm{SiO}_{2}$ peak and the $\mathrm{Si}$ peak. In the estimation, cylindrical shape with the height the same as the diameter was assumed [31], and 2.5 and $2.9 \mathrm{~nm}$ were taken as the mean free path of photoelectrons in $\mathrm{Si}$ and $\mathrm{SiO}_{2}$ [32], respectively, and 1.1 for the ratio of the cross-sections in $\mathrm{Si}$ and $\mathrm{SiO}_{2}$ [33]. The $\mathrm{SiO}_{2}$ thickness was estimated to be 0.7 $\mathrm{nm}$ for spectrum a, $2.6 \mathrm{~nm}$ for spectrum $\mathrm{b}$, and $4.8 \mathrm{~nm}$ for spectrum c. Therefore, it can be concluded that the $\mathrm{SiO}_{2}$ thickness increases when the hydrogen generation reaction proceeds, and that the reaction stops when the 4.8

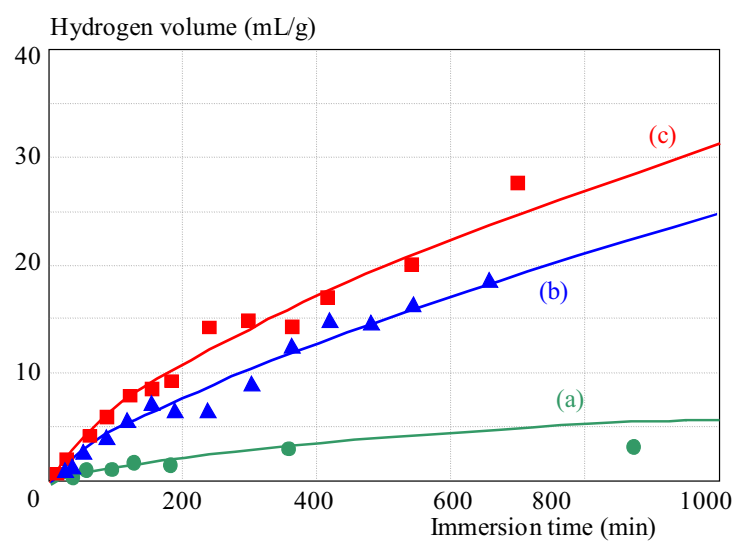

Fig. 4. Generated hydrogen volume vs the reaction time for HFetched $1 \mathrm{~g}$ Si nanopowder in ultrapure water at room temperature formed with the following methods: (a) - without milling, (b) one-step beads milling, (c) - two-step beads milling

$\mathrm{nm} \mathrm{SiO}_{2}$ layer is formed because it acts as a complete migration barrier for $\mathrm{OH}^{-}$ions. It is noted that in the case of thermal oxidation of $\mathrm{Si}$, much higher temperature (ie $800 \approx 900^{\circ} \mathrm{C}$ ) is needed because diffusion of oxygen molecules through growing $\mathrm{SiO}_{2}$ proceeds only at high temperatures [34]. On the other hand, migration of $\mathrm{OH}^{-}$ ions through $\mathrm{SiO}_{2}$ is likely to proceed much more easily for the following reasons: (i) $-\mathrm{OH}^{-}$ions are smaller than $\mathrm{O}_{2}$, (ii) - electrical field in $\mathrm{SiO}_{2}$ induced by adsorption of $\mathrm{OH}^{-}$ions enhances inner migration of $\mathrm{OH}^{-}$ions [35].

Figure 4 shows the hydrogen volume generated by the reaction of Si powder and Si nanopowder produced with one-step and two-step beads milling methods with ultrapure water. The hydrogen generation rate for $5 \mathrm{~m}$ Si powder (ie, without milling) (plot a) was the lowest, and the generated hydrogen volume monotonically increased with time. Generated hydrogen volume also increased monotonically with time for $\mathrm{Si}$ nanopowder fabricated with one-step beads milling (plot b) and two-step beads milling (plot c). The hydrogen generation rate for two-step beads milling was $1.3 \sim 1.4$ times higher than that for one-step beads milling. This ratio is in reasonable agreement with the ratio in the surface area estimated from the average diameter of Si nanopowder, ie 1.7. The Si nanopowder aggregated and the surface area of agglomerates of two-step beads milled Si nanopowder was only 1.1 times that of one-step beads milled Si nanopoweder of $20 \mathrm{~m}^{2} / \mathrm{g}$. Therefore, it can be concluded that the reaction rate for hydrogen generation is strongly dependent on the crystallite size but almost independent of the size of agglomerates.

$\mathrm{Si}$ and the product, $\mathrm{SiO}_{2}$, formed by the reaction of $\mathrm{Si}$ with water, are both nonpoisonous, and therefore, Si can be taken for internal hydrogen generation. As is evident from Fig. 2, Si reacts with water under alkaline environment. Therefore, no reaction proceeds in stomach due to gastric acids while $\mathrm{Si}$ nanopowder reacts with water in bowels with alkaline environment due to pancreatic juice with $\mathrm{pH}$ 8.5. Hydrogen molecules are effectively absorbed 
in bowels, circulate in the whole body, and react with hydroxyl radicals which cause various diseases such as cancers and Parkinson's disease [21-23].

Table 1. Maximum hydrogen generation rate and total hydrogen volume generated from $1 \mathrm{~g}$ Si nanopower by the reaction with the strong alkaline solutions

\begin{tabular}{lcc} 
Solution condition & $\begin{array}{c}\text { Maximum } \\
\text { generation rate } \\
(\mathrm{ml} / \mathrm{g} / \mathrm{min})\end{array}$ & $\begin{array}{c}\text { Generated } \\
\text { total volume } \\
(\mathrm{ml} / \mathrm{g})\end{array}$ \\
\hline $\mathrm{pH} 12.9$ at $\mathrm{RT}$ & 122 & 971 \\
$\mathrm{pH} 13.0$ at $50{ }^{\circ} \mathrm{C}$ & 580 & 1442 \\
$\mathrm{pH} 13.9$ at $\mathrm{RT}$ & 351 & 1589 \\
\hline
\end{tabular}

Table 1 shows the total hydrogen volume generated by the reaction of $\mathrm{Si}$ nanopowder with strong alkaline solutions with $\mathrm{pH}$ higher than 12.9 , and the maximum hydrogen generation rate. In this case, Si nanopowder was fabricated from Si swarf, ie industrial waste. With strong alkaline solutions, the hydrogen generation rates became much higher than those for solutions with the neutral $\mathrm{pH}$ region. The hydrogen generation rate strongly depended on the temperature. The generated hydrogen volume depended on $\mathrm{pH}$ of the solutions, ie $1590 \mathrm{~mL}$ for $\mathrm{pH} 13.9$ and $970 \mathrm{~mL}$ for $\mathrm{pH} 12.9$. The $\mathrm{pH}$ dependence can be explained by the following reaction mechanism: In the presence of high concentration $\mathrm{OH}^{-}$ions, Si nanopowder directly reacts with $\mathrm{OH}^{-}$ions, leading to generation of hydrogen and formation of soluble $\mathrm{SiO}_{2}(\mathrm{OH})_{2}{ }^{2-}$ ions:

$$
\mathrm{Si}+2 \mathrm{OH}^{-}+2 \mathrm{H}_{2} \mathrm{O} \rightarrow \mathrm{SiO}_{2}(\mathrm{OH})_{2}{ }^{2-}+2 \mathrm{H}_{2} .
$$

For reaction (4), no $\mathrm{SiO}_{2}$ layer is formed, and therefore, in cases where reaction (4) is dominant, $1600 \mathrm{~mL}$ hydrogen is produced from $1 \mathrm{~g} \mathrm{Si}$ nanopowder. For the reaction with strong alkaline solutions, $\mathrm{pH}$ of the solutions decreased by hydrogen generation, in good agreement with reaction (4) in which $\mathrm{OH}^{-}$ions were consumed for hydrogen generation. For the reaction with alkaline solutions having $\mathrm{pH} \sim 13$, reactions (3) and (4) simultaneously proceed, and when $\sim 4.8 \mathrm{~nm} \mathrm{SiO}_{2}$ is formed by reaction (3), hydrogen evolution stops.

It should be noted that the generated hydrogen volume and the hydrogen generation rate are considerably high for the reaction with strong alkaline solutions, and Si nanopowder can be fabricated from Si swarf using the simple beads milling method. Therefore, this technology is expected be applied to fuel cells. For hydrogen generation using efficient photocatalysts [18], on the other hand, more than 1 year is necessary for generating of $1600 \mathrm{~mL}$ hydrogen using $1 \mathrm{~g}$ photocatalyst.

\subsection{Photoluminescence from Si nanopowder}

Si swarf was used for fabrication of Si nanopowder for PL emission. Figure 5 shows the photographs of blue PLemitting Si nanopowder (Fig. 5a), green PL-emitting Si nanopowder (Fig. 5b), and hexane solvent (Fig. 5c). The photographs were taken under black light $(365 \mathrm{~nm})$ irradiation. The intensity of blue PL was much higher than that of green PL. Green PL-emitting Si nanopowder was obtained after HF etching of Si nanopowder and immersion in ethanol, while blue PL-emitting Si nanopowder after immersion in hexane without HF etching.

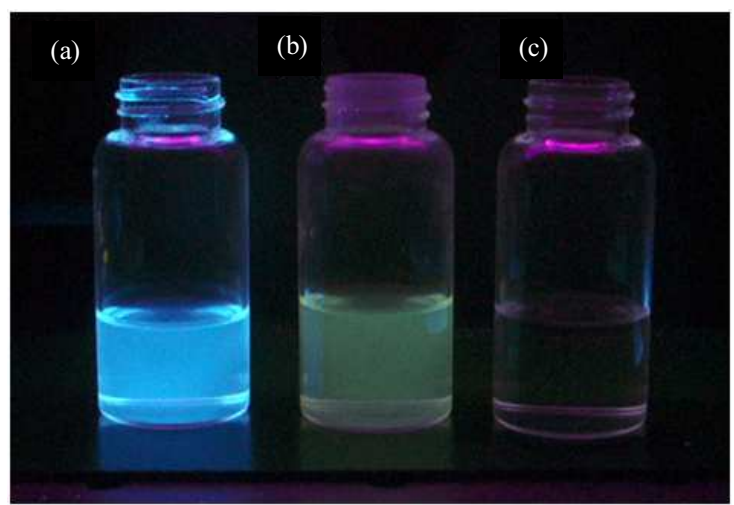

Fig. 5. Photographs of PL emitting Si nanopowder under black light irradiation: (a) - Si nanopowder in hexane, (b) - HF-etched Si nanopowder in ethanol, (c) - hexane solvent without Si nanopowder

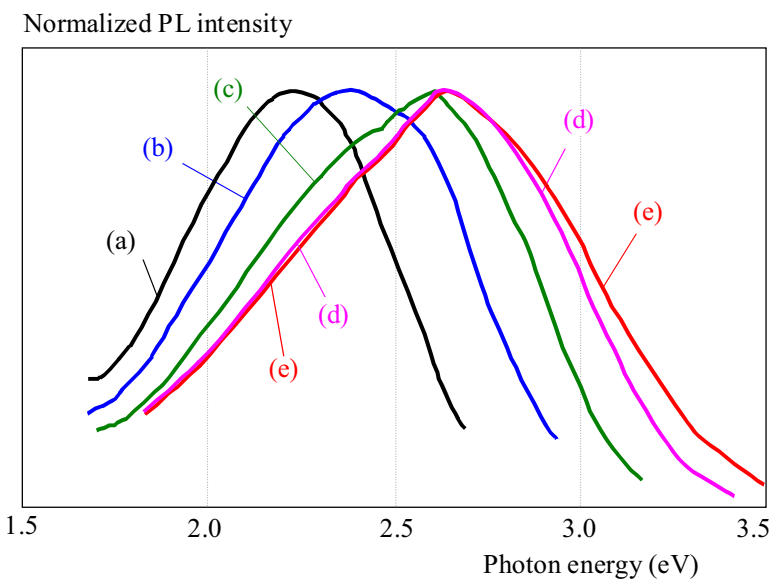

Fig. 6. PL spectra for HF-etched $\mathrm{Si}$ nanopowder in ethanol observed under UV irradiation with the following excitation energies: (a) $-2.76 \mathrm{eV},(\mathrm{b})-3.02 \mathrm{eV},(\mathrm{c})-3.26 \mathrm{eV},(\mathrm{d})-3.54 \mathrm{eV},(\mathrm{e})-3.76$ $\mathrm{eV}$

Figure 6 shows the PL spectra for green PL-emitting Si nanopowder. Only a broad peak was observed, and the PL peak showed a blue shift with an increase in the excitation light energy. The width of the PL peak increased with the excitation energy. From these results, the PL peak is attributed to band-to-band transition of Si nanopowder whose band-gap is widen by the quantum confinement effect $[4,5]$. The blue shift results from excitation of smaller Si nanopowder with higher band-gap energies, followed by PL emission. The increase in the PL peak width arises from an increase in the portion of Si nanopowder which can be excited by the higher energy excitation light.

Figure 7 compares the PL spectra for Si nanopowder fabricated with one-step beads milling (spectrum a) 


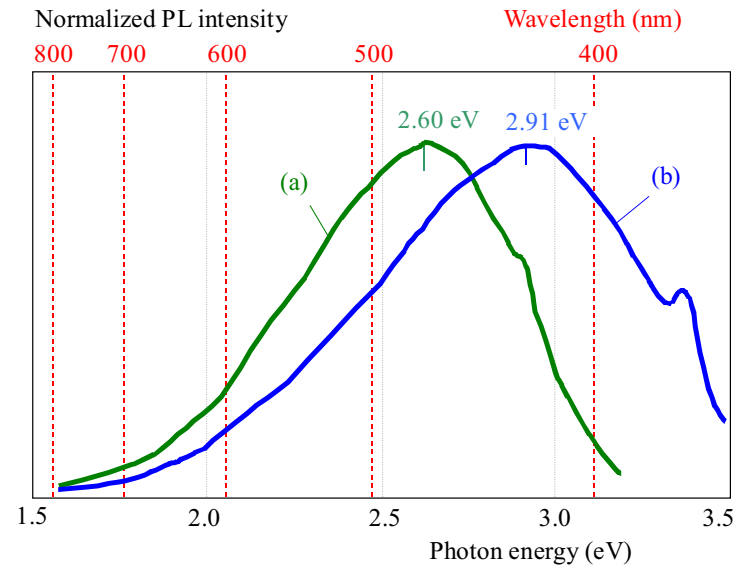

Fig. 7. PL spectra for HF-etched Si nanopowder in ethanol fabricated with the following methods: (a) - one-step beads milling method, (b) - two-step beads milling method

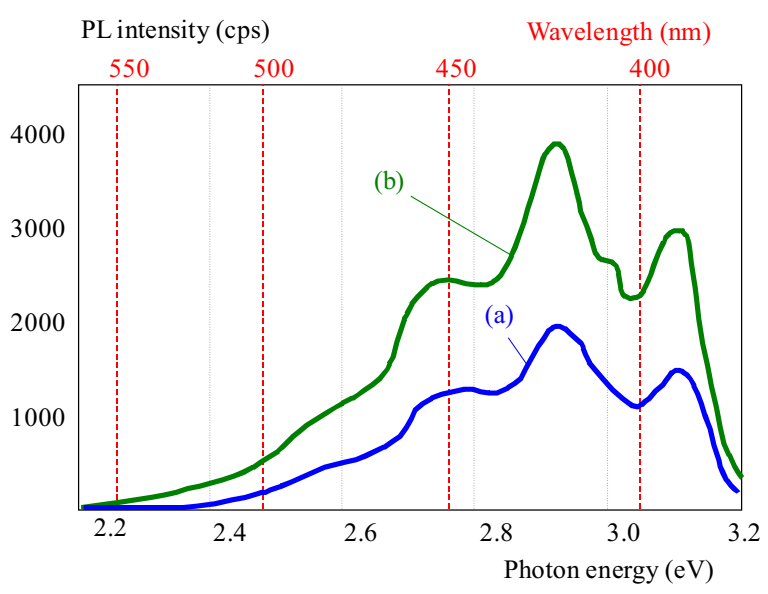

Fig. 9. PL spectra for Si nanopowder without HF etching in hexane fabricated with the following methods: (a) - one-step beads milling method, (b) - two-step beads milling method, [14].

and two-step beads milling (spectrum b). One-step beads milled Si nanopowder exhibited the PL peak with the peak maximum at $2.60 \mathrm{eV}$ while $\mathrm{Si}$ nanopowder fabricated with the two-step beads milling method showed the peak maximum at $2.91 \mathrm{eV}$. These results demonstrate that green PL results from band-to-band transition, and thus, the smaller the size of Si nanopowder, the higher the band-gap energy, leading to higher energy PL [36,37].

Figure 8 shows the PL spectra for blue PL-emitting Si nanopowder. The PL spectra possessed peaked structure, and their energies didn't depend on the excitation light energy. The PL spectra were almost the same as that for 9,10-dimethylantracene (DMA), and therefore, blue PL is most likely to arise from DMA impurity adsorbed on $\mathrm{Si}$ nanopowder. The peaked structure is attributable to vibronic bands which involve the total symmetric breathing mode of DMA in the electronic ground state [38], and the bands at $3.14,3.34$, and $3.53 \mathrm{eV}$ are assigned as the $(0,2)$, $(0,1)$, and $(0,0)$ transitions, respectively where $(m, n)$ denotes the transition from the $\mathrm{m}$-th vibrational state of the electronic excited state to the $\mathrm{n}$-th vibrational state of

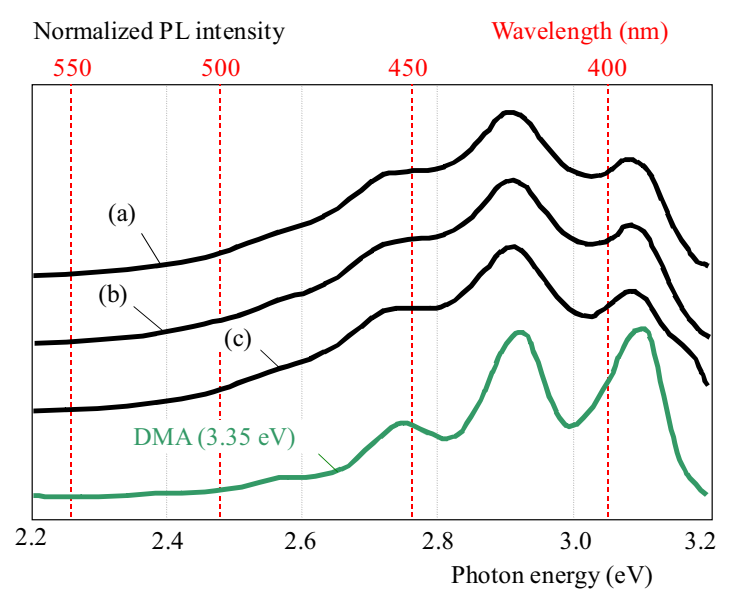

Fig. 8. PL spectra for Si nanopowder without HF etching in hexane observed under UV irradiation with the following excitation energies: (a) $-3.17 \mathrm{eV}$, (b) $-3.36 \mathrm{eV}$, (c) $-3.50 \mathrm{eV}$, the bottom spectrum is for a DMA solution without Si nanopowder

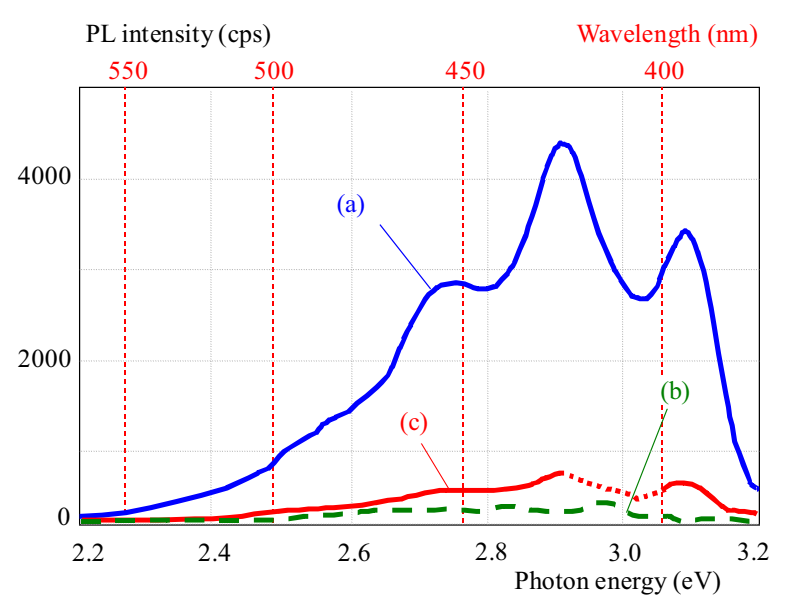

Fig. 10. PL spectra of Si nanopowder without HF etching in hexane observed under the conditions: (a) - before addition of an HF plus $\mathrm{HNO}_{3}$ solution, (b) - after addition of an $\mathrm{HF}$ plus $\mathrm{HNO}_{3}$ solution,

(c) - hexane solvent concentrated by 39 times by boiling, [14]

the electronic ground state. The result that the energies of these peaks are independent of the excitation light energy clearly shows single source of PL emission, ie adsorbed DMA. The PL spectra for DMA-adsorbed Si nanopowder possessed slightly broader structure than that of free DMA, due to overlapping of the vibronic bands on the broad background arising from band-to-band transition of Si nanopowder.

Figure 9 compares the PL spectra for green PLemitting Si nanopowder produced with one-step beads milling (spectrum a) and two-step beads milling (spectrum b). Both the spectra possessed nearly identical structure with the vibronic bands at the same energies, clearly showing that blue PL did not arise from Si nanopowder. The PL intensity for two-step beads milled Si nanopowder was higher than that for one-step beads milled Si nanopowder, and the quantum efficiency was estimated to be $9 \%$ for one-step beads milled Si nanopowder and $15 \%$ for two-step beads milled Si nanopowder. This result may indicate that DMA is more efficiently 
adsorbed on smaller Si nanopowder, most probably due to larger total surface area.

When a small amount of an $\mathrm{HF}$ plus $\mathrm{HNO}_{3}$ solution was added to blue light-emitting Si nanopowder in hexane, the PL peaks disappeared almost completely (spectrum b in Fig. 10). Because $\mathrm{HF}$ plus $\mathrm{HNO}_{3}$ solutions dissolve $\mathrm{Si}$, this result clearly shows that the PL intensity is greatly enhanced by adsorption of DMA on Si nanopowder.

Hexane solvent was concentrated by 39 times by evaporation, and its PL spectrum is shown in Fig. 10(c). Although he PL intensity was very low, the vibronic bands were observed at the same energies as those for free DMA. From the comparison of the PL intensity of DMAadsorbed Si nanopowder to that for spectrum c, the enhancement factor is estimated to be $\sim 3000$. The PL enhancement may result from the following reasons: (i) increase in the dipole moment of DMA due to adsorption on Si nanopowder, (ii) - enhancement of wave function of DMA by adsorption (eg surface resonance state). More detailed studies are necessary to clarify the reason for the PL enhancement.

\section{Conclusion}

Si nanopowder was fabricated by use of the beads milling method, and applied to hydrogen generation source and PL emitting material. Detailed investigation leads to the following results and conclusion:

(1) Si nanopowder reacts with water in the neutral $\mathrm{pH}$ region, generating hydrogen. $\mathrm{OH}^{-}$ions act as catalyst, and therefore, the hydrogen generation rate greatly increases with $\mathrm{pH}$, while $\mathrm{pH}$ of the solutions isn't changed by the hydrogen generation reaction.

(2) The hydrogen evolution rate and the hydrogen volume generated by the reaction with neutral water strongly depend on the crystallite size but don't on the size of aggregates.

(3) Reaction of Si nanopowder with strong alkaline solutions (eg pH13.9 KOH solutions) can generate 1600 $\mathrm{mL}$ hydrogen, ie the theoretical limit. In this case, $\mathrm{pH}$ of the solutions decreases after the reaction because $\mathrm{OH}^{-}$ ions are consumed to form soluble $\mathrm{SiO}_{2}(\mathrm{OH})_{2}{ }^{2-}$ ions.

(4) HF-etched Si nanopowder in ethanol exhibits green PL. The PL peak shows blue shifts by a decrease in the size of Si nanopowder and also by an increase in the excitation light energy. The green PL is attributed to band-to-band transition of Si nanopowder.

(5) Si nanopowder without $\mathrm{HF}$ etching in hexane shows blue PL spectra with peaked structure. The PL energies don't depend on the size of Si nanopowder and the excitation light energy. The peaked structure is attributed vibronic bands of DMA molecules adsorbed on Si nanopowder.

(6) The intensity of blue PL is enhanced by $\sim 3000$ by adsorption on Si nanopowder.

\section{Acknowledgements}

This work is partially supported by COI Stream.

\section{REFERENCES}

[1] F. Erogbogbo, T. Lin, P. M. Tucciarone, K. M. Lajoie, L. Lai, G. D. Patki, P. N. Prasad and M. T. Swihart, "On-Demand Hydrogen Generation using Nanosilicaon, Splitting Water without Light, Heat, or Electricity",Nano Lett. 13, (2013) 451-456.

[2] G. Conibeer, Applications of Si Nanoparticles Photovoltaic Solar Cells Silicon Nanocrystals, PAVESI, L. TURAN, R. ed., Silicon Nanocrytals. Weinheim", Wiley-VCH Verlag GmbH \& Co.KGaA; 2010.p.555-561.

[3] S. Yang, W. Li, B. Cao, H. Zeng and W. Cai, "Origin of Blue Emission from Silicon Nanoparticles", Direct Transition and Interface Recombination, J.Phys Chem.C 115, (2011) 21056-21062.

[4] L. Mangolini, "Synthesis, Properties, and Applicaion of Silicon Nanocrystals", J.Vac.Sci.Technol. B31 (2013) 020801-1-29.

[5] O. Gusev, A. N. Poddubny, A. A. Prokofiev and I. N. Yassievich, "Light Emission from Silicon Nanocrystals", Semiconductors 47 (2013) 183-202.

[6] W. T. Leach, J. -H. Zhu and J. G. Ekerdt, "Thermal Desorption Effects Chemical Vapor Deposition of Silicon Nanoparticles", J.Crystal Growth 243 (2002) 30-40.

[7] K. Watanabe, K. Sawada, M. Koshiba, M. Fujii and S. Hayashi, "Photoluminescence Decay-Dynamics of Si Nanoparticles Prepared by Pulsed Laser Ablation", Appl.Surf.Sci. 197-198 (2002) 635-638.

[8] S.-M. Liu, M. SATO, S. Kobayashi and K. Kimura, "Synthesis of Silicon Nanowires and Nanoparticles by Arc-Discharge Water", Chem.Commun.(2005) 4690-4692.

[9] D. Arquier, G. Calleja, G. Cerveau and R. J. P. Corriu, "A New Solution Route for the Synthesis of Silicon Nanoparticles Presenting Different Surface Substituents", C.R.Chimie 10 (2007) 795-802.

10] C.-S. Yang, R. A. Bley, S. M. Kauzlarich, H. W. H. Lee and G. R. Delgado, "Synthesis of Alkyl-Terminated Silicon Nanoclusters by a Solution Route", Y J.Am.Chem.Soc., 121 (1999) 5191-5195.

[11] M. Maeda, K. Imaura, T. Matsumoto and H. Kobayashi, "Fabrication of Si Nanoparticles from Si swarf and application to solar cells", Appl.Surf.Sci. 312 (2014) 39-42.

[12] K. Imamura, K. Kimura, S. Fujie and H. Kobauyashi, "Hydrogen Generation from Water Using Si Nanopowder Fabricated from Si Swarf", J. Nanoparticle Research, 18 (2016) 116-1-7.

[13] K. Kimura, T. Matsumoto and H. Kobayashi, to be submitted.

[14] T. Matsumoto, M. Maeda and H. Kobayashi, "Photoluminescence Enhancement of Adsorbed Species on Si, Nanoscale", Res.Lett. 11 (2016) 7-1-6.

[15] T. Matsumoto, M. Maeda, J. Furukawa, W.-B. Kim and H. Kobayashi, "Si Nanoparticles fabricated from Si swarf by Photochemical Etching Method Matsumoto" J.Nanopart.Res., 16, (2014) 2240-1-7.

[16] T. Sun, E. Liu, X. Liang, X. Hu and J. Fan, "Enhanced Hydrogen Evolution from Water Splitting Using Fe-Ni Codoped and Ag Deposited Anatase TiO2 Synthesized by Solvothermal Method", J.Appl.Surf.Sci. 347 (2015) 696-703.

[17] M. Y. Xie, K. Y. Su, R. J. Peng-wu, M. Chaveli and W. C. Chang, "Hydrogen Production by Photocatalytic Water-Splitting on Pt-Doped TiO2-ZnO under Visible Light", Taiwan Inst. Chem. Eng., 70 (2017) 161-167.

[18] X. Liu, L. Zhao, K. Domen and K. Takanabe, "Photocatalytic Hydrogen Production Using Visible-Light-Responsive Ta3N5 Photocatalyst Supported on Monodisperse Spherical $\mathrm{SiO}_{2}$ Particles", Mater.Res.Bull. 49 (2014) 58-65. 
[19] S. Litvinenko, S. Alekseev, V. Lysenko, A. Venturello, F. Geobaldo, L. Gulina, G. Kuznetsov, V. Tolstoy, V. Skryshevsky, E. Garrone and BARBIER, "Hydrogen Production from Nano-Porous Si Powder Formed by Stain Etching", Inter. J. Hyd. Ener., 35 (2010) 6773-6778.

[20] C. Zhan, P. K. Chu, D. Ren, Y. Xin, K. Huo, Y. Zou and N. K. Huang, "Release of Hydrogen during Transformation from Porous Silicon to Silicon Oxide at Normal Temperature", Inter.J.Hyd.Ener. 36 (2011) 4513-4517.

[21] C. P. Maurizi, "Alzheimer's Disease", oles for Mitochondrial Damage, the Hydroxyl Radical, and Cerebrospinal Fluid Deficiency of Melatonin", Med.Hypotheses. 57 (2001) 156-160.

[22] Y. Ihara, M. Chuda, S. Kuroda and T. Hayabata, "Hydroxyl Radical and Superoxide Dismutase Blood of Patients with Parkinson's Disease: Relationship to Clinical Data", J. Neurol. Sci. 170 (1999) 90-95.

[23] D. Charvin, P. Vanhoutte, C. Pages and E. CABOCHE. J. Borrelli, "Unraveling a Role for Dopamine Huntington's Disease: The Dual Role of Reactive Oxygen Species and D2 Receptor Simulation", J.Proc.Natl.Acad.Sci. 102 (2005) 12218-12223.

[24] Z. Kang, Y. Liu and S. -T Lee, "Small-sized Silicon Nanoparticles", New Nanolights and Nanocatalysts, 3, (2011) 777-791.

[25] Y. Zhu, H. Wang and P. P. Ong, "Evidence of Photoluminescence Related to Subsurface Si-O-Si Bonds Sputtered Silicon Nanoparticles", Solid State Commun. 116 (2000) 427-429.

[26] M. Inada, H. Nakagawa, I. Umezu and A. Sugimura, "Effect of Hydrogenation on Photoluminescnence of Si Nanoparticles Formed by Pulsed Laser Ablation", Mater.Sci.Eng. B101 (2003) 283-285.

[27] T. Matsumoto, M. Maeda and H. Kobayashi, "Photoluminescence Enhancement of Adsorbed Species on SI", Nanoscale Res. Lett., 11 (2016) 7-1-6.

[28] Y. V. Ryabchikov, S. A. Alekseev, V. Lysenko, G. Bremond and J. M. Bluet, "Photoluminescence of Silicon Nanoparticles Chemically Modified by Alkyl Groups and Dispersed Low-Polar Liquids", J.Nanopart.Res. 15 (2013) 1535-1-9.

[29] F. J. Himpsel, F. R. Mcfeely, A. YARMOFF, J. A. Taleb-Ibrahi$\mathrm{mi}$ and G. Hollinger, "Microscopic Structure of the $\mathrm{SiO}_{2} / \mathrm{Si}$ Interface", Phys.Rev. B 38 (1988) 6084-6096.

[30] H. Kobayashi, T. Ishida, Y. Nakato and H. Tsubomura, "Mechanism of Carrier Transport Highly Efficient Solar Cells Having Indium Tin Oxide/Si Junctions", J.Appl.Phys. 69 (1991) 1736-1743.

[31] O. Renault, R. Marlier, N. T. Barrett, E. Martinez, T. Baron, M. Gely and B. D. Saivo, "Modeling the XPS Si 2p Core-Level Intensities of Silicon Nanocrystals for Determination of Oxide Shell Thickness", Surf.Interface Anal. 38 (2006) 486-488.

[32] H. Kobayashi, O. Asuha-maeda, T. Takahashi and H. Iwasa, "Nitiric Acid Oxidation of Si to Form Ultrathin Silicon Dioxide Layers with a Low Leakage Current Density", J.Appl.Phys. 94 (2003) 7328-7335.

[33] M. F. Hochella Jr. and A. H. Carim, "A Reassessment of Electron Escape Depths Silicon and Thermally Grown Silicon Dioxide Thin Films", Surf.Sci. 197 (1988) L260-L268.

[34] B. E. Deal and A. S. Grove, "General Relationship for the Thermal Oxidation of Silicon", J.Appl.Phys. 36 (1965) 3770-3778.

[35] D. D. Eley and P. R. Wilkinson, "Adsorption and Oxide Formation on Aluminum Films", Proc.R.Soc.London A 254 (1960) 327-342.

[36] S. Azuma and S. Sato, "Size Regulation of Si Nanoparticles through Photon-Enhanced Chemical Etching", Chem.Lett. 40 (2011) 1294-1296.

[37] J. D. Holmes, K. J. Ziegler, R. C. Doty, L. E. Pell, K. P. Johnston and B. A. Korgel, "Highly Luminescent Silicon Nanocrystals with Discrete Optical Transitions", J.Am.Chem.Soc. 123 (2001) 3743-3748.

[38] K. Ohno, "Simple Calculations of Franck-Condon Factors for Electronic Transition Bands of Polyacenes", Chem.Phys.Lett.53 (1978) 571-577.
Received 23 April 2017

Yuki Kobayashi (Specially appointed Researcher) received his BA degree in Business Administration from Kwansei Gakuin University, Nishinomiya, Japan, in 2012. From 2012 to 2013, he was an analyst in Consumer Insight Office of Takasago International Corporation, Tokyo, Japan where he engaged in development of flavors and fragrances. Since 2013, he has been a Specially Appointed Researcher in the Institute of Science and Industrial Research, Osaka University, Osaka, Japan. His research interest is Si nanoparticle to generate hydrogen and its application to the medicine and pharmacy.

Kentaro Imamura (Assist Prof, Sci, PhD) received his MSc and PhD degrees in chemistry from Osaka University, Osaka, Japan, in 2008 and 2012, respectively. From 2008 to 2012, he was a Researcher in Corporate Research \& Development group of Sharp Corporation, Nara, Japan where he engaged in development of LCD devices. Since 2012, he has been an Assistant professor in the Institute of Science and Industrial Research, Osaka University, Osaka, Japan. His research interests are Si solar cells, especially new chemical methods to improve conversion efficiencies such as surface structure chemical transfer (SSCT) method for achievement of ultralow reflectance and nitric acid oxidation of silicon (NAOS) method for surface passivation, and fabrication and application of nano-Si materials

Taketoshi Matsumoto (Assoc Prof, Sci, PhD) received his MSc and PhD degrees electronic chemistry from Tokyo Institute of Technology, Yokohama, Japan in 2001. From 2000 to 2002, he was a research fellow of the Japan Society for the Promotion of Science. Since 2001, he was a Postdoctoral Research Associate in University of Southern California, CA, US. Since 2003, he was a Lecturer in University of Tsukuba, Tsukuba, Japan. Since 2004, he was a Research Associate in Institute for Molecular Science, Okazaki, Japan. Since 2007 and 2014, he has been an Assistant and Associate Professor, respectively, in the Institute of Scientific and Industrial Research, Osaka University, Osaka, Japan. He is interested in energy related nano-materials and devices such as TFTs, LSI, luminous materials, sealed permanent memories, solar cells and $\mathrm{Li}$ ion batteries.

Hikaru Kobayashi received the BE degree in chemistry from Osaka University, Osaka, Japan, in 1979 and the ME, and $\mathrm{PhD}$ degrees in chemistry from Kyoto University, Kyoto, Japan, in 1981, and 1984, respectively. From 1984 to 1986, he was a Postdoctoral Research Associate in Department of Physics and Astronomy, University of Pennsylvania, PA. From 1986-1987, he was a Researcher in Matsushita Electronics Corporation Kyoto Research Center, Kyoto, Japan where he was engaged in LSI wiring and gate oxide technology. From 1987 to 1990, he was a Research Associate in Department of Chemistry, Faculty of Engineering Science, Osaka University where he was engaged in research on silicon solar cells, surface and interface science of silicon, and low temperature silicon oxidation. From 1990 to 1998, he has been an Associate Professor in Department of Chemistry, Faculty of Engineering Science, Osaka University. He is currently a Professor in the Institute of Scientific and Industrial Research, Osaka University. He has been engaged in research on silicon solar cells, low temperature $\mathrm{Si}$ oxidation, and fabrication of $\mathrm{Si}$ nanopowder and its application to hydrogen generation material, anode material in $\mathrm{Li}$ ion batteries, and luminescent material. 